\title{
Laparoscopic totally transhiatal diverticulectomy of giant epiphrenic oesophageal diverticulum
}

\author{
Nik Ritza Kosai ${ }^{1}$, Hardip Singh Gendeh ${ }^{1, *}$, Jing Liang $\mathrm{Ho}^{1}$, Yi Rong Chiew ${ }^{1}$, Mustafa Mohamed \\ Taher $^{1}$, Paul Anthony Sutton ${ }^{2}$ \\ ${ }^{1}$ Department of Surgery, Universiti Kebangsaan Malaysia Medical Centre (UKMMC), Kuala Lumpur, Malaysia \\ 2Institute of Translational Medicine, University of Liverpool, Liverpool, United Kingdom
}

\begin{abstract}
Epiphrenic diverticulum is a rare form of oesophageal diverticulum involving the distal part of the oesophagus. We report the case of a 51 year-old lady who initially presented with nocturnal coughing and volume reflux. Clinical examination was unremarkable as were initial routine blood investigations. A plain chest radiograph, barium swallow, and computed tomography were performed which demonstrated features of a large oesophageal diverticulum. Oesophagogastroduodenoscopy revealed a wide neck, single diverticulum within the lower third of the oesophagus. Oesophageal manometry undertaken revealed normal oesophageal peristalsis and lower oesophageal sphincter pressure. An elective total transhiatal laparoscopic diverticulectomy with Heller's cardiomyotomy and fundoplication was performed with good symptomatic improvement. The patient made an uneventful recovery and remains symptom free two years postoperatively.
\end{abstract}

Key Words: Fundoplication, diverticulectomy, endoscopic, cardiomyotomy; epiphrenic

\section{Introduction}

Epiphrenic diverticulum is a rare form of oesophageal diverticulum which involves the distal third of the oesophagus within $10 \mathrm{~cm}$ of the oesophagogastric junction (1). Most patients with oesophageal diverticulum are asymptomatic, however a small proportion may complain of anorexia, epigastric pain, dyspepsia, nocturnal cough and weight loss. As a result there are frequent difficulties and delays in reaching a diagnosis.

We report a case of an epiphrenic diverticulum presenting in a lady with a history of reflux symptoms which was successfully treated with a diverticulectomy combined with Heller's cardiomyotomy and fundoplication via an exclusively laparoscopic transhiatal approach.

\section{Case report}

A 51 year-old fit and well female presented with progressively worsening post-prandial dyspepsia and as acid brash with vomiting for the past six months. The vomitus contained fluid and undigested food particles. Physical examination and routine blood investigations revealed no abnormalities.
A plain chest radiograph, barium swallow (Figure 1), and computed tomography performed demonstrated features of a large single oesophageal diverticulum. Oesophagogastroduodenoscopy (OGD) revealed a wide neck, single diverticulum within the lower third of the oesophagus (Figures 2 and 3). Oesophageal manometry was subsequently undertaken which revealed normal lower oesophageal sphincter (LOS) pressure and peristalsis. On the basis of these investigations, the patient underwent an elective laparoscopic diverticulectomy combined with Heller's cardiomyotomy and fundoplication.

The patient was placed on a reverse trendelenburg lithiotomy position. Two twelve millimeter and five millimeter ports were inserted, and a twelve millimeter mercury pressured carbon dioxide pneumoperitoneum was created. The liver was retracted superiorly with a Nathanson retractor. The distal oesophagus was mobilized 360degrees to identify the diverticulum site, confirmed with intraoperative OGD. A wide-neck giant epiphrenic diverticulum was identified posterolaterally at $28 \mathrm{~cm}$ from the incisors. Upon fully mobilizing the diverticulum, its wide neck was transected with an EndoGIATM (COVIDIEN) sixty millimeters tanned coloured stapler cartridge

*Corresponding Author: Dr. Hardip Singh Gendeh, Department of Surgery University Kebangsaan Malaysia Medical Centre Jalan Yaacob Latif 56000 Cheras Kuala Lumpur, Malaysia, Tel: +6-03-91456202, Fax: +6-03-91456684, E-mail: hardip88@gmail.com 


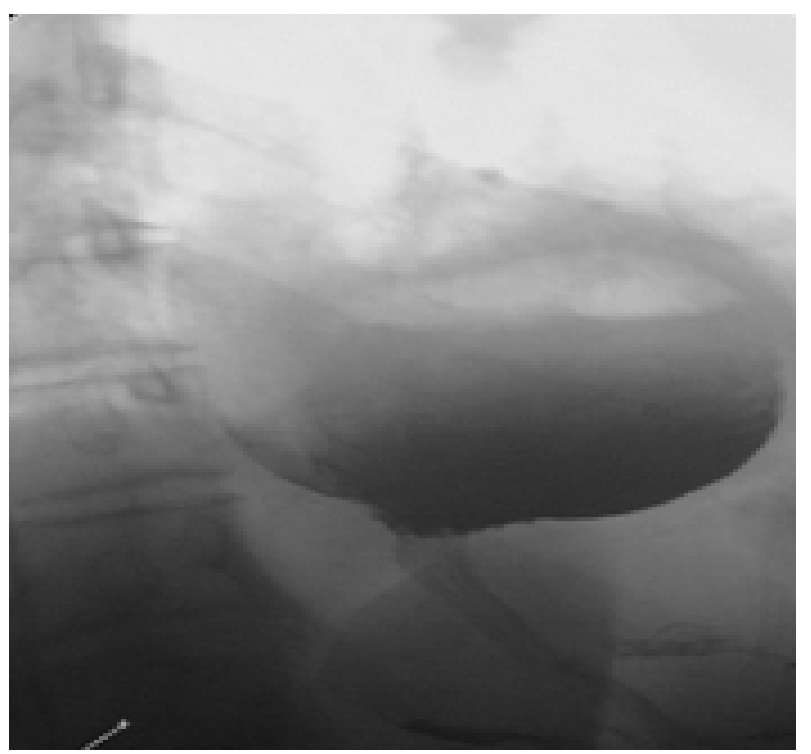

Fig. 1. Large Epiphrenic diverticulum on barium swallow.

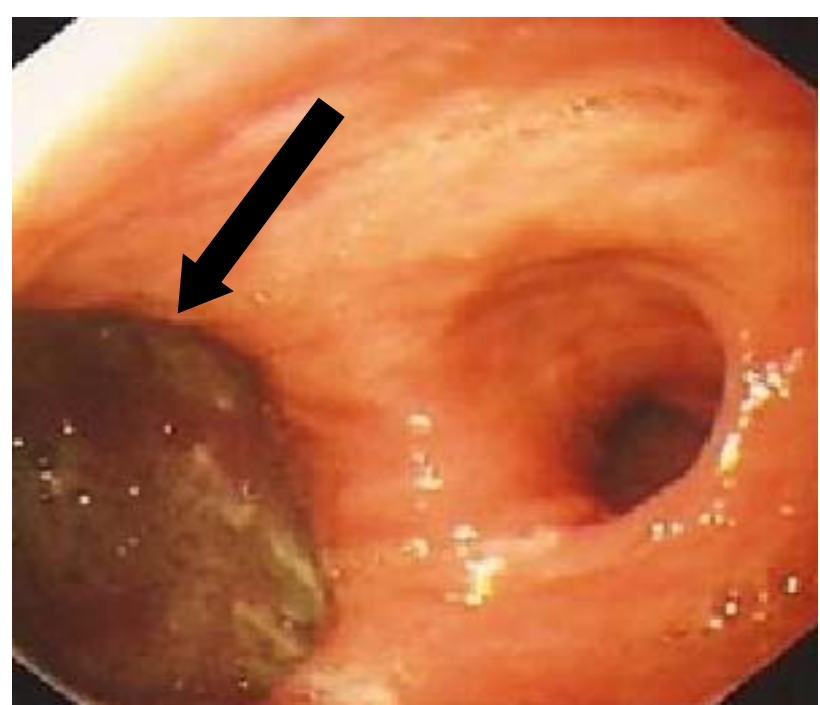

Fig. 2. OGD revealing a broad based giant epiphrenic oesophageal diverticulum (arrow) with oesophageal lumen on the right.

without over sewing the stapler line (Figures 4 and 5). Subsequently, a cardiomyotomy was performed three centimeters distal to the gastroesophageal junction with a proximal extension to the superior neck of the diverticulum (Figure 6). A 180 degrees anterior Dor fundoplication was performed (Figure 7). An epiphrenic oesophageal diverticulum measuring $7 \times 5 \times 3 \mathrm{~cm}$ in dimensions was resected (Figure 8). Air and methylene blue test showed no leak. The procedure was complicated by a simple pneumothorax which was treated successfully by insertion of a right intercostal chest tube.

Post-operatively the patient made an unremarkable recovery. She was commenced on

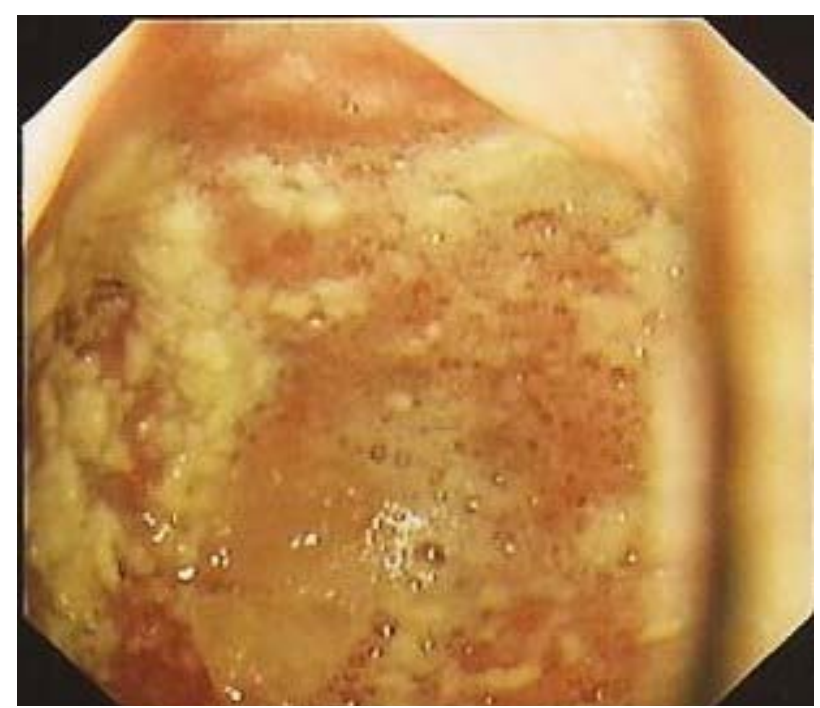

Fig. 3. OGD showing internal mucosa of broad base diverticulum with refluxed gastric contents.

soft diet following a gastrograffin study on day two which demonstrated no leak (Figure 9). The chest tube was removed on day three and the patient was discharged home on post-operative day six. She remains asymptomatic at two years post-operatively.

\section{Discussion}

Most patients with oesophageal diverticulum are asymptomatic, however a small proportion may complain of anorexia, epigastric pain, dyspepsia, nocturnal cough and weight loss (2). Life threatening conditions such as aspiration pneumonia and malignancy are rare, however can 


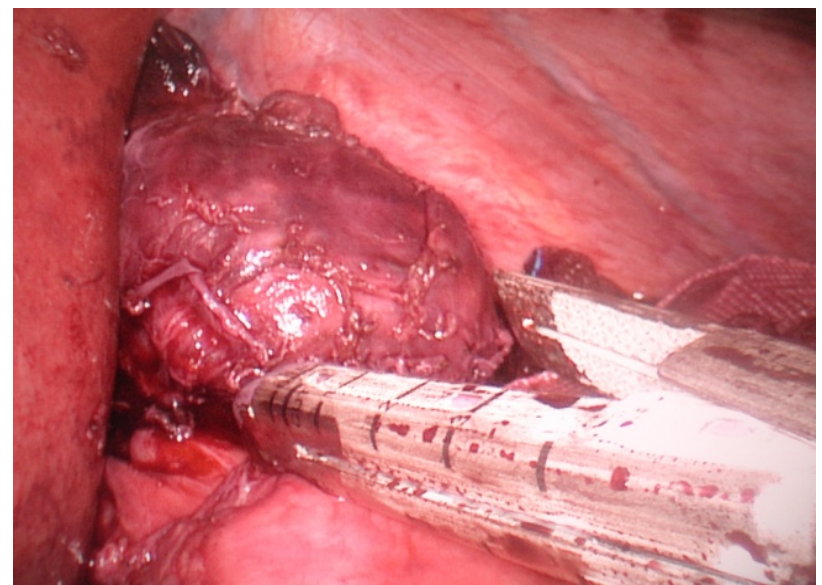

Fig. 4. Giant epiphrenic oesophageal diverticulum with laparoscopic stapler.

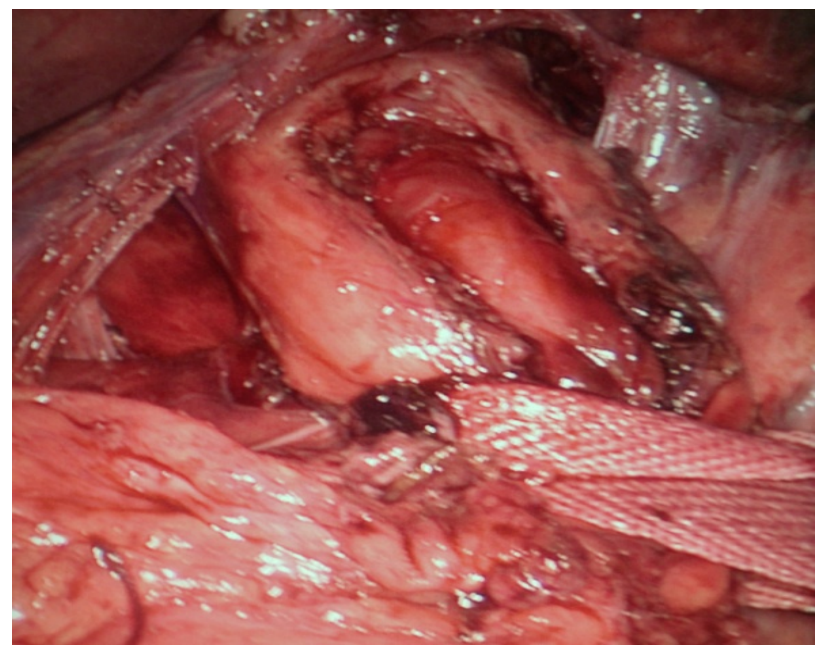

Fig. 6. Heller's oesophageal myotomy performed.

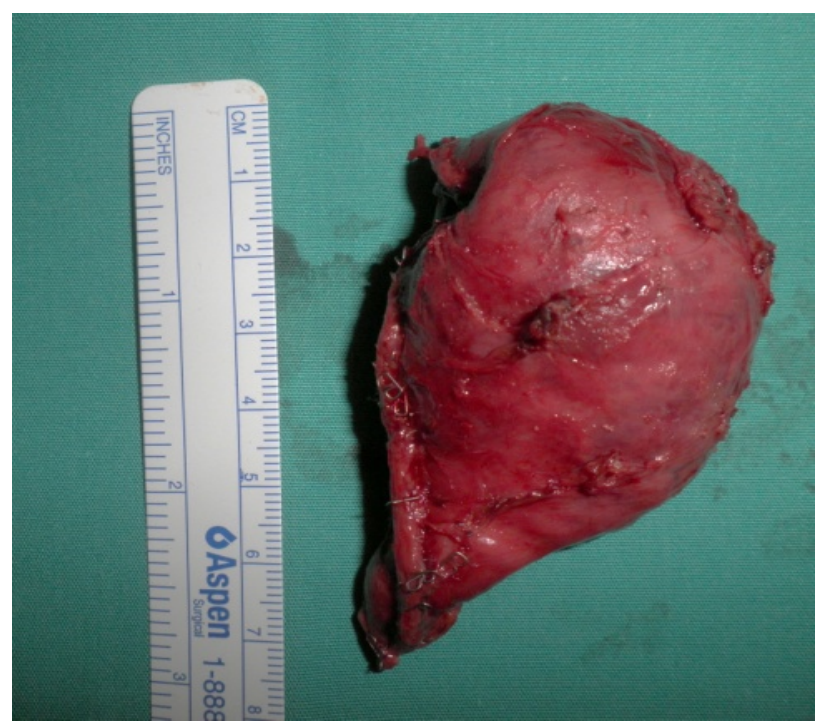

Fig. 8. Resected stapled giant epiphrenic oesophageal diverticulum measuring $7 \times 5 \times 3 \mathrm{~cm}$ in dimensions.

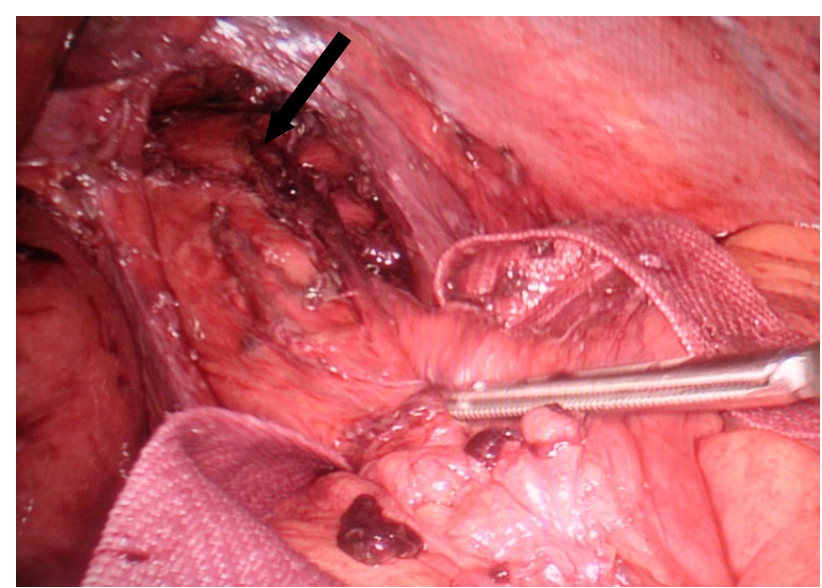

Fig. 5. Stapler line (arrow) visible following transection of diverticulum.

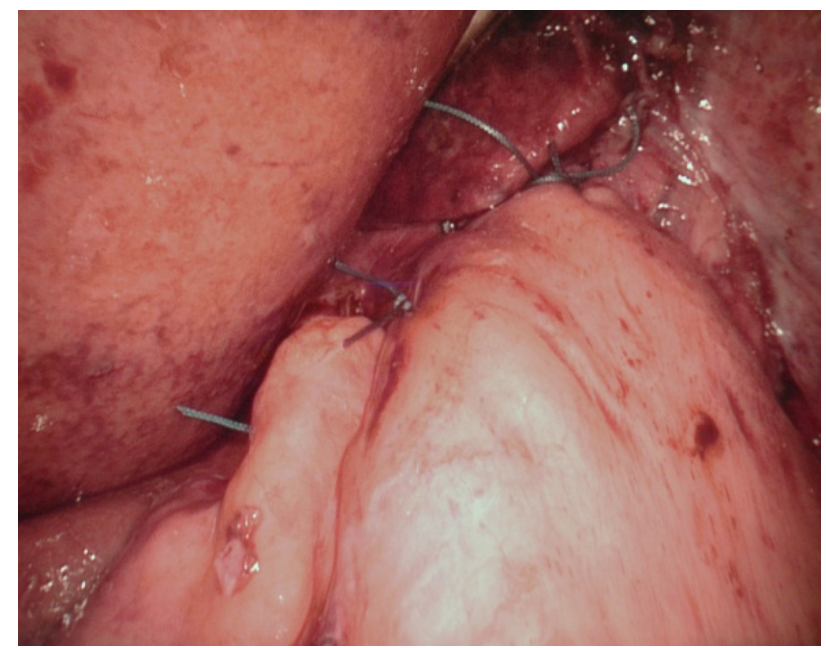

Fig. 7. An anterior dor partial fundoplication performed.

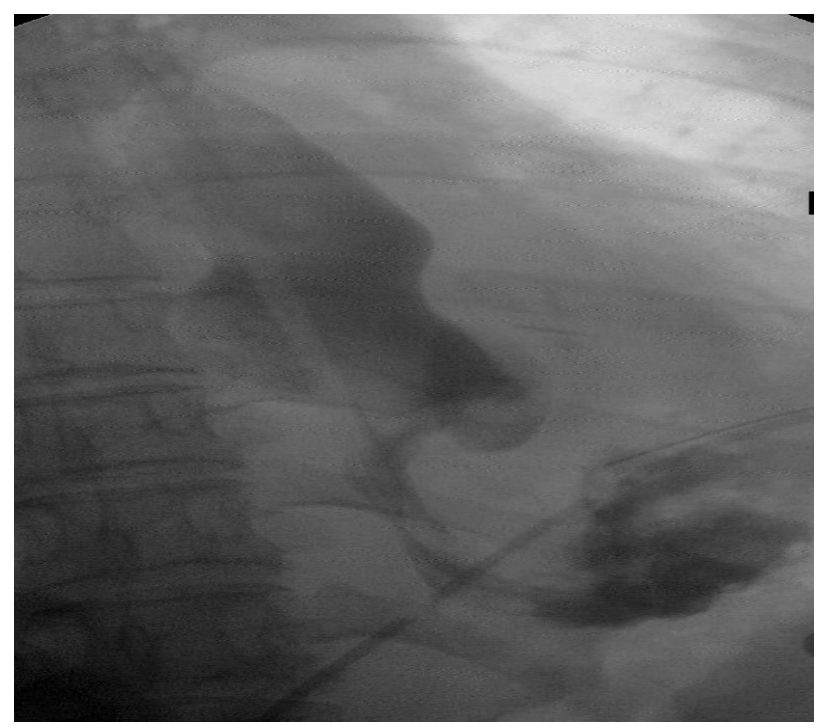

Fig. 9. Post -operative Gastrograffin study showing no leak. 
occur with delayed diagnosis (2). The condition is normally apparent on a plain chest radiograph, OGD, barium swallow and computed tomography scan. Oesophageal manometry should be performed to confirm the presence of oesophageal motility disorder, commonly the underlying pathophysiology of epiphrenic diverticulum.

Surgical intervention should be considered in large or symptomatic diverticulae. Such intervention can be accomplished either by an open or minimally invasive procedure, the latter of which may either be video assisted thoracoscopy (VAT) or laparoscopy or in combination. The procedure includes diverticulectomy with or without cardiomyotomy and fundoplication (1). However the absence of cardiomyotomy with fundoplication may lead to increased risks of reccurance and leak (1). It has been demonstrated that a minimally invasive approach to epiphrenic diverticulum results in reduced mortality, morbidity and length of stay with no significant difference in symptomatic improvement compared with open procedures (1).

Numerous comparisons have been made between VAT and laparascopic approaches. Despite the fact that the VAT can offer a clearer visualisation of the entire diverticulum in patients with giant diverticulum, the use of double-lumen endotracheal tubes, single lung ventilation, and postoperative chest tubes makes this approach more complex with an associated increase in morbidity (3). It is also shown there are no significant differences in reported outcomes between the thoracoscopic and laparoscopic approaches in patients with small (five $\mathrm{cm}$ or less) diverticulae (4). Although the thoracoscopic, or combined thoracoscopic/laparoscopic approach, is thought to be preferable in patients with higher or giant diverticulum $(1,2)$, our case demonstrates that in giant epiphrenic diverticulae surgical intervention can be safely completed with a totally transhiatal laparoscopic approach without any post operative complications and leak.
In cases of epiphrenic diverticulae it is thought that myotomy is not mandatory and should selectively performed when LOS pressure is below normal (5). Although LOS pressure was normal on preoperative oesophageal manometry, given the nature of the diverticulum we did not believe that oesophageal motility disorder could be excluded as the underlying cause, given its intermittent nature. Since the underlying pathophysiology of epiphrenic diverticulum is most commonly oesophageal motility disorder, we would suggest that myotomy is considered in all cases of epiphrenic diverticulum.

Laparoscopic diverticulectomy is preferred to open surgery for the management of epiphrenic diverticulum, resulting in decreased length of stay, morbidity and mortality. It is a safer and more feasible alternative to thoracoscopic surgery whilst offering comparable outcomes. A cardiomyotomy with fundoplication should be considered insimilar presentation with likely underlying motility disorder, even in the presence of normal LOS pressure on oesophageal manometry.

\section{References}

1. Kilic A, Schuchert MJ, Awais O, Luketich JD, Landreneau RJ. Surgical management of epiphrenic diverticula in the minimally invasive era. JSLS 2009; 13: 160-164.

2. Clark SC, Norton SA, Jeyasingham K, Ridley PD. Oesophageal epiphrenic diverticulum: an unusual presentation and review. Ann R Coll Surg Engl 1995; 77: 342-345.

3. Sharp KW, Khaitan L, Scholz S, Holzman MD, Richards WO. 100 Consecutive Minimally Invasive Heller Myotomies: Lessons Learned. Ann Surg 2002; 235: 631-639.

4. Matthews BD, Nelms CD, Lohr CE, et al. Minimally invasive management of epiphrenic esophageal diverticula; Am Surg 2003; 69: 465470.

5. Hudspeth DA, Thorne MT, Conroy R, Pennell TC. Management of epiphrenic esophageal diverticula. A fifteen-year experience. Am Surgeon 1993; 59: 40-42. 